Original

\title{
Resultados del uso de la técnica de Mitrofanoff en pacientes con disfunción neurógena de la vejiga: Análisis de la experiencia en el Hospital Universitario San Vicente de Paul
}

\author{
María E. Arango Rave*, Luis F. Lince Varela*, Catalina Salazar Sanín**, \\ Francisco C. Hoyos Figueroa* Sara N. Hurtado*, Juan C. Rendón Isaza** \\ *Departamento de Cirugía Pediátrica. ${ }^{* *}$ Cirugía General. Universidad de Antioquia. Colombia
}

\begin{abstract}
Resumen
Introducción: El cuidado de los niños con vejiga neurogénica, debe ser integral, multidisciplinario y busca preservar la función renal, alcanzar continencia urinaria y fecal, logrando que el paciente sea una persona autosuficiente y útil a la sociedad.

Métodos: Describimos el resultado del uso de la técnica de Mitrofanoff en el manejo de pacientes con disfunción vesical neurógena, utilizada en el HUSVP desde 1998 al 2003, y las condiciones actuales de los pacientes con respecto a su enfermedad y tratamiento. Se realizó un estudio observacional descriptivo, retrospectivo 1998-2002 y prospectivo durante el 2003, analizando una serie de casos.

Resultados: Fueron intervenidos 41 pacientes con vejiga neurogénica. Edad promedio 10.2 años; seguimiento promedio 27,2 meses; enfermedad de base más frecuente mielomeningocele (46,3\%); vejiga disinérgica en 46,3\%, esfínter hipotónico en $41,5 \%$. Se realizo aumento vesical al $68,3 \%$, utilizándose ileon en 71,4\%, y alguna intervención sobre el cuello vesical al $51,2 \%$.

Se hizo una derivación tipo Mitrofanoff al 95,1\% con continencia completa en $70,8 \%$, incontinencia completa en $14.6 \%$ e incontinencia

ocasional en 14,6\%. En 31,7\% se realizó un Malone con adecuada continencia fecal en 90,2\%. De los estomas el 19\% presentaron estenosis y el 21,9\% presentaron escape de orina. Se encuentran socialmente adaptados $80 \%$ de los pacientes.

Conclusiones: Los estomas continentes cateterizables son útiles para tratar la incontinencia urinaria y fecal. Los conductos realizados con ileon tuvieron mayores complicaciones que los elaborados con apéndice, por lo cual el apéndice debe ser el tejido de elección para realizar las derivaciones, cuando esté disponible.
\end{abstract}

Palabras clave: Vejiga neurogénica. Mitrofanoff. Estoma continente cateterizable. Incontinencia urinaria.

\section{Outcomes the Mitrofanoff technique in the management of patients with neurogenic bladder: the experience in the San Vicente de Paul Universitary Hospital}

\section{Abstract}

Introduction: The care for children with neurogenic bladder, should be integral, multidisciplinary look to preserve renal function and to accomplish urinary and fecal continence, achieving that the patient becomes self-sufficient and useful to society.

Methods: The result of the use of the Mitrofanoff technique for the treatment of patients with neurogenic dysfunction bladder used in the HUSVP 1998- 2003, and the current condition of the patients with respect to their illness and treatment are described in this article. A descriptive retrospective study 1998- 2002 and a prospective study during 2003 were done, in which a series of cases were analyzed.

Results: 41 patients had surgery. Average age 10.2 years; average follow-up time 27.2 months; the most frequent illness was myelomeningocele (46.3\%) and 46.3\% had dysinergic bladder. Bladder augmentation was performed on $63.3 \%$, of which $71.4 \%$ were constructed with ileum. A surgical intervention of the bladder neck was done on $51.2 \%$.

A continent conduct (Mitrofanoff) was performed on $95.1 \%$ of the patients with complete continence 70.4\%, complete incontinence $14.6 \%$ and occasional incontinence 14.6\%. 31.7\% had Malone surgery with adequate fecal management on 90.2\%. $19 \%$ of the stomas presented stenosis and $21.9 \%$ presented urine leakage. $80 \%$ reached appropriate social adaptation. Conclusions: The continent catheterizable stomas are useful for the treatment of urinary and fecal incontinence. The conducts constructed with ileum had more complications than the conducts done with cecal appendix, which is why the appendix is the choice tissue to perform the continent catheterizable stomas, as long as it is available.

Keywords: Neurogenic bladder. Mitrofanoff. Continent catheterizable stoma. Urinary incontinence. 
T a función del tracto urinario inferior depende de Aa coordinación neuromuscular de la vejiga, la uretra y el músculo estriado responsable del mecanismo esfinteriano. Un trastorno neurológico o muscular en estas estructuras ocasiona una disfunción miccional.

La vejiga neurogénica que se produce por un trastorno del control neurológico de la continencia y la micción, comprende todos los problemas de micción cuyo origen está en el sistema nervioso central. La primera causa de vejiga neurogénica disfuncional en niños es la espina bífida oculta o disrafismo espinal, sin embargo, también puede ser producida por agenesia sacra, tumores, enfermedades inflamatorias que afectan la médula espinal o por trauma raquimedular. Es indispensable llevar a cabo estudios urodinámicos e imagenológicos para determinar la función del tracto urinario, tanto alto como bajo con el fin de establecer un plan de tratamiento para cada paciente ${ }^{1}$, y posteriormente realizar un seguimiento adecuado ${ }^{3}$. En vejiga neurogénica los hallazgos urodinámicos determinan básicamente: vejiga disinérgica, vejiga sinérgica y vejiga con ausencia de actividad.

El manejo de los niños con vejiga neurogénica debe ser integral, multidisciplinario y tiene tres objetivos fundamentales: Preservar la función renal, buscar la continencia urinaria y fecal, y lograr que el paciente llegue a ser una persona autosuficiente y útil a la sociedad.

El tratamiento busca que el paciente alcance la continencia urinaria y que el tracto urinario superior permanezca protegido de las alteraciones progresivas que se producen como consecuencia del aumento de las presiones en el tracto urinario inferior. Para lograr esto es necesario realizar intervenciones dirigidas a convertir una vejiga de baja capacidad y/o alta presión en un reservorio con una capacidad adecuada a una baja presión, que permita una evacuación fácil y completa, y un esfinter hipotónico en un mecanismo competente que no permita la salida involuntaria de orina. También debería conservarse la capacidad de micción espontánea por la uretra, lo cual es más difícil de lograr. El manejo de la vejiga neurogénica debe comenzar tan pronto como se realice el diagnóstico con el fin de evitar el deterioro progresivo del tracto urinario superior. El comienzo temprano del cateterismo limpio intermitente ${ }^{4}$, cada tres horas en el periodo neonatal, es seguro y bien tolerado, permite un manejo a largo plazo de la vejiga neurogénica y evita el deterioro del tracto urinario superior, protegiendo al niño mientras éste alcanza la edad suficiente para enfrentar un procedimiento quirúrgico definitivo, cuando éste es necesario. Los procedimientos quirúrgicos que normalmente se realizan son: el aumento vesical ${ }^{2,5}$, los estomas continentes dentro de los que se encuentra el Mitrofanoff ${ }^{6-10}$ y el tubo de Monti $^{11,12}$, esfínteres artificiales ${ }^{13}$, cincha ure$\operatorname{tral}^{2,13}$, inyección submucosa del cuello vesical ${ }^{2,13}$, reconstrucción del cuello vesical ${ }^{2,13}$ y cerclaje ure$\mathrm{tral}^{2}$. El tratamiento quirúrgico puede ser complementado con terapia farmacológica, la cual consiste en la administración de medicamentos anticolinérgicos que producen relajación del detrusor ${ }^{4}$.

La mayoría de los pacientes con neuropatías causantes de vejiga neurogénica presentan también alteraciones de la función colónica y ano-rectal que provocan incontinencia fecal, por lo cual en algunos pacientes se realizan procedimientos que permitan la realización de enemas anterógrados como es el Malone ${ }^{14-21}$.

La principal complicación que se presenta con los estomas es la estenosis ${ }^{7,19,22,23}$, por lo cual se han diseñado diferentes técnicas que utilizan colgajos para evitarla o corregirla ${ }^{24}$.

El resultado final de la técnica de Mitrofanoff puede evaluarse en términos de continencia urinaria, ausencia de complicaciones del estoma, reintervenciones quirúrgicas por complicaciones y protección del tracto urinario superior. En general, puede decirse que los resultados en cuanto a continencia son buenos, aunque en las series con seguimiento más largo se reporta una alta frecuencia de revisiones quirúrgicas del conducto, por filtración y estenosis $^{7,19,22-, 24}$.

En el Hospital Universitario San Vicente de Paúl, de Medellín, Colombia, desde el año 1998 se han ofrecido alternativas de tratamiento quirúrgico a los niños con vejiga y colon neurogénicos.

Esta investigación tiene el propósito de describir el tipo de tratamiento utilizado durante un período de 8 y 1/2 años, en los pacientes con vejiga neurogénica, que consultaron al Servicio de Cirugía Pediátrica del HUSVP y de la Universidad de Antioquia, así como su evolución clínica.

\section{METODOS Y PACIENTES}

Se realizó un estudio observacional descriptivo, retrospectivo y prospectivo, en el cual se analizó una 
serie de casos. La información se recolectó por medio de una encuesta, a partir de la revisión de historias clínicas complementándose con una llamada telefónica al paciente o a la persona responsable de su cuidado, con la cual se obtuvo los datos acerca de su condición actual.

Se evaluaron los registros clínicos de todos los pacientes intervenidos quirúrgicamente por vejiga neurogénica en el HUSVP, que cumplian criterios de inclusión.

Las preguntas de la entrevista telefónica que evalúan los resultados de la continencia urinaria y fecal, y la adaptación social, se consignaron en el formulario de recolección de datos y son las siguientes:

\begin{tabular}{lcccc} 
20. Continencia urinaria: & \multicolumn{1}{c}{ 1. Completa } & \multicolumn{2}{c}{ 2. Incompleta } \\
& 3. Incontinente & 4. ND \\
21. Continencia fecal: & 1. Sí & 2. No & 3. ND \\
22. Estenosis estomal: & 1. Sí & 2. No & 3. ND \\
23. Filtración fecal estomal: & 1. Sí & 2. No & 3. ND \\
27. Aceptación del cateterismo: & 1. Sí & 2. No & 3. ND \\
28. Adaptación social: & 1. Sí & 2. No & 3. ND
\end{tabular}

\section{CRITERIOS DE INCLUSION}

Todos los pacientes pediátricos, menores de 12 años que hubieran sido tratados quirúrgicamente para vejiga neurogénica en HUSVP entre el 1 de enero de 1998 y el 31 de mayo del 2006, y cuyos registros clínicos estuviesen disponibles.

\section{PLAN DE ANALISIS}

Se empleo el software estadístico SPSS 10. Para el análisis descrito, las variables cualitativas se informaron como proporciones y las cuantitativas como promedios o mediana, y medidas de dispersión como la desviación estándar y los rangos. Se realizó un análisis exploratorio univariado comparando las variables cualitativas independientes con la variable dependiente, para esto se empleó la prueba exacta de Ficher. Para el análisis comparativo de variables cuantitativas se empleó la prueba de U de Mann-Whitney.

\section{RESULTADOS}

En total se logró obtener adecuada documentación de 41 registros clínicos de pacientes, 23 de sexo masculino y 18 de sexo femenino; no hubo ninguna relación entre el sexo y la enfermedad de base. La edad promedio al momento de la cirugía fue de 10.2 años (rango de 1-20 años).
El tiempo promedio de seguimiento fue de 27.2 meses (rango de 1-72 meses). La enfermedad de base más frecuente fue el mielomeningocele presente en 19 pacientes (46.3\%), seguido de otras malformaciones espinales en 5 pacientes $(12,2 \%)$, valvas de la uretra posterior en 5 pacientes (12,2\%) (Tabla 1).

Tabla 1. Enfermedades de base

\begin{tabular}{lc}
\hline Enfermedad de base & Número de pacientes (\%) \\
\hline Mielomeningocele & $19(46,3)$ \\
Trauma medular & $1(2,4)$ \\
Malformaciones congénitas* & $4(9,8)$ \\
Valvas uretra posterior & $5(12,2)$ \\
Síndrome de Ochoa & $4(9,8)$ \\
Otras** $^{*}$ & $8(19,5)$ \\
\hline
\end{tabular}

*Malformaciones congénitas incluyen: extrofia de vejiga (2 pacientes), megauretra corta (1 paciente), agenesia de uretra (un paciente). **Otras: malformaciones espinales (5 pacientes), vejiga neurogénica primaria (un paciente), trauma de uretra (un paciente) y neuroblastoma (un paciente).

$\mathrm{El}$ valor promedio de la creatinina fue $0,8 \mathrm{mg} / \mathrm{dL}$ (rango 0,2-2,7 mg/dL), los valores más altos se encontraron en pacientes con diagnóstico previo de valvas de la uretra posterior.

En los estudios urodinámicos se encontró que 19 pacientes $(46,3 \%)$, tenían una vejiga hipertónica, de los cuales 10 tenían mielomeningocele y 2 otra malformación espinal; 13 pacientes $(31,7 \%)$ tenían vejiga con ausencia de actividad y solo 3 $(7,3 \%)$ tenían una vejiga normal; en 6 pacientes no se obtuvo el dato. En cuanto al esfínter, 17 pacientes tenian esfinter con ausencia de actividad $(41,5 \%)$ de los cuales 13 tenían diagnóstico de mielomeningocele; en 6 pacientes no se obtuvo el dato. En 14 pacientes se halló además una disinergia detrusor-esfínter.

El 85\% de los pacientes presentó uno o más episodios de infección urinaria antes de la cirugía. Ninguna de las patologías de base se relacionó con una mayor frecuencia de infección urinaria.

Se presentaron alteraciones estructurales del tracto urinario en 20 pacientes $(48,8 \%)$. No hubo ninguna relación entre las alteraciones estructurales del tracto urinario y la patología de base.

Se efectuó un aumento vesical en 28 pacientes $(68,3 \%)$, con vejigas de poca capacidad, de los cuales 15 tenían como diagnóstico un mielomeningocele; 4 de ellos tenían valvas de la uretra posterior; 3 
un sindrome urofacial, y 4 otras malformaciones congénitas. El tejido utilizado para el aumento vesical fue el ileon en 20 pacientes $(71,4 \%)$, en 7 (25\%) se utilizo el uréter y en uno (3,6\%) el colon. No hubo relación entre el tipo de tejido utilizado y la patología de base (Fig. 1).

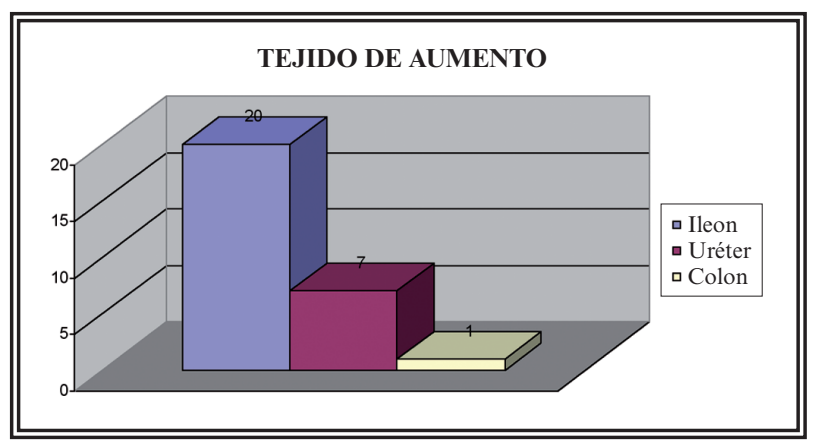

FIGURA 1. Tejido usado en el aumento vesical.

En 21 pacientes $(51,2 \%)$ se realizó algunos procedimientos sobre el cuello vesical como una cincha uretral, un cerclaje del cuello vesical o un alargamiento del mismo. No se utilizo ningún esfínter artificial. El 66.6\% de estos pacientes (14 de 21) tenían diagnostico de mielomenigocele.

La elaboración de un conducto vesicocutáneo continente cateterizable tipo Mitrofanoff se efectuó en 39 pacientes (95,1\%); en 25 de los 39 (64,1\%) el tejido utilizado fue el apéndice, seguido de íleon (tubo de Monti) en 10 y uréter en 4 pacientes. La elección del tejido a utilizar se hizo de acuerdo con la presencia o no del apéndice cecal, o porque se usara el apéndice para la construcción de un conducto que permitiera la administración de enemas anterógrados (cirugía de Malone), por esta razón la mayoría de las derivaciones de tipo Mitrofanoff realizadas con íleon (tubo de Monti) se hicieron en niños con diagnóstico de mielomeningocele (8 de 10).

El procedimiento de Malone se llevó a cabo en 13 pacientes $(31,7 \%)$, en 12 de ellos se utilizó el apéndice cecal y en un paciente se usó un colgajo de ciego. De estos pacientes, 9 tenían diagnostico de mielomeningocele, 3 de malformaciones espinales y 1 de trauma medular. El tiempo promedio de seguimiento de estos pacientes fue de 28.4 meses.

La continencia urinaria tanto por el conducto cateterizable de Mitrofanoff como por la uretra fue definida como completa en 29 pacientes $(70,8 \%)$; incompleta, o con escape de orina ocasional, en 6 pacientes $(14,6 \%)$ e incontinente en 6 pacientes (14,6\%) (Fig. 2).

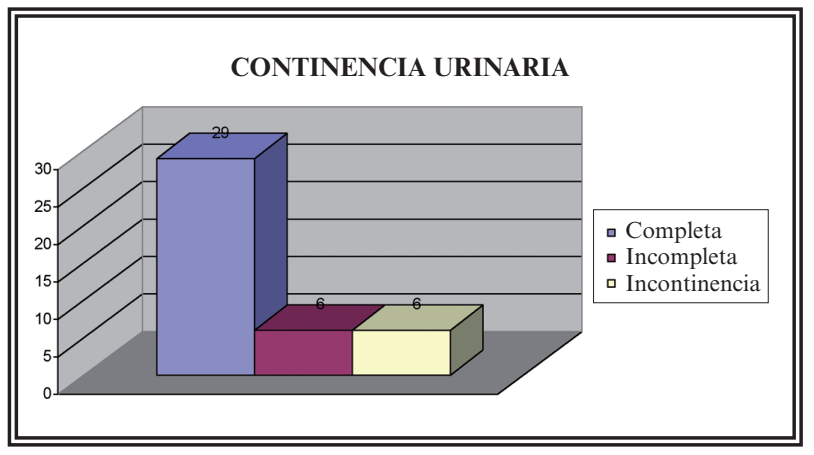

FIGURA 2. Resultados en continencia urinaria.

El tiempo promedio de seguimiento de los pacientes con continencia completa fue 24.6 meses, con continencia incompleta fue 34.2 meses y con incontinencia fue 32.6 meses. El grado de continencia no se relaciono con la edad a la cual se realizo la cirugía inicial.

Al relacionar el tipo de tejido utilizado para la construcción del conducto cateterizable y la continencia urinaria se encontró, que se logró continencia urinaria completa en 20 de los pacientes (80\%) en los cuales se utilizo apéndice, en 5 (50\%) en los que se utilizo íleon y en $3(75 \%)$ en los que se utilizo uréter. Estenosis del estoma del conducto cateterizable se presento en 8 pacientes, 3 (12\%) de los estomas realizados con apéndice, 3 (30\%) con íleon y $2(50 \%)$ con uréter. El tiempo promedio de seguimiento de los pacientes que presentaron estenosis del estoma fue 38.7 meses. Igualmente 9 pacientes presentaron filtración de orina por el conducto cateterizable, 4 (16\%) fueron realizados con apéndice, 4 (40\%) con íleon y $1(25 \%)$ con uréter.

De los 41 pacientes $37(90,2 \%)$ tienen adecuada continencia fecal, los 4 restantes tienen como diagnóstico de base mielomeningocele; solo un paciente de los 13 a los cuales se les realizo el procedimiento de Malone no logró buen manejo fecal. El tiempo promedio de seguimiento de los pacientes que no lograron continencia fecal fue 25,5 meses y de los que lograron continencia fecal fue 27,3 meses. Ninguno de los pacientes presentó filtración fecal por el estoma. Solo un paciente presentó estenosis del Malone, porque el paciente nunca aceptó cateterizarse para realizar los enemas anterógrados.

Se realizaron 46 procedimientos adicionales en 18 pacientes $(44 \%)$, un paciente tuvo 15 procedimientos. A 16 pacientes se les realizo revisión del estoma, a 2 revisión vesical y a 4 pacientes procedimientos sobre el cuello vesical. 
Las causas por las cuales se realizaron estas intervenciones fueron estenosis del meato (9), filtración de orina (9), incontinencia uretral (2), necrosis del conducto (1), perdida del injerto (1), obstrucción del conducto (1) y prolapso de la mucosa (1). Un paciente con Malone realizado con apéndice requirió un procedimiento adicional por estenosis del estoma. El 50\% de los pacientes que requirieron procedimientos adicionales tenían diagnostico de mielomeningocele.

Actualmente 40 pacientes se realizan cateterismo vesical intermitente por la uretra o a través del conducto cateterizable de Mitrofanoff y un paciente no permite los cateterismos. Se cateterizan en forma adecuada $36(87,8 \%)$ y hay 4 pacientes con inconvenientes para el cateterismo (uno tiene continencia urinaria por el Mitrofanoff y por la uretra, uno tiene escape moderado de orina por el Mitrofanoff y los otros dos son incontinentes). No hay relación entre el tiempo de seguimiento, el tipo de vejiga, el tejido usado para el conducto de Mitrofanoff o la edad al momento de la cirugía, y la aceptación del cateterismo.

Actualmente 33 de los pacientes (80\%) se encuentran con adecuada adaptación social en términos de interacción con el medio y escolaridad. De estos, 26 están secos entre los cateterismos (25 por el Mitrofanoff y uno por la uretra), 5 tienen algún grado de incontinencia uretral o por el Mitrofanoff y los dos restantes son incontinentes por uretra.

El $100 \%$ de los pacientes con valvas uretrales posteriores o síndrome urofacial tienen adecuada actividad social, mientras que el $49 \%$ de los pacientes con vejiga neurogénica secundaria a defectos del tubo neural presentan alguna dificultad relacionada con la escolaridad o la adaptación social.

\section{DISCUSIÓN}

El tratamiento exitoso de la incontinencia urinaria y fecal secundaria a trastornos neuropáticos, puede mejorar la calidad de vida de los niños, posibilitándoles socialización y bienestar. El desarrollo de la técnica del conducto vesicocutáneo cateterizable descrita por Mitrofanoff ha sido la principal contribución en el logro del control adecuado de la continencia urinaria $^{25} \mathrm{y}$ ha sido de gran importancia en especial en los pacientes de bajos niveles socioeconómicos y educacionales $^{34}$. Desde el momento en que fue expuesto este principio, el apéndice se ha constituido en el tejido de elección para la elaboración del conducto. Sin embargo, en algunos casos el apéndice no está disponible o se necesita para otros fines como la construcción del conducto de Malone. Yang en 1993 y
Monti en $1997^{11,15}$ desarrollan una técnica alternativa para crear el conducto cateterizable vesicocutáneo a partir de un segmento de intestino, que se convirtió en la mejor opción para su elaboración con un elemento diferente al apéndice, este procedimiento es sencillo, genera poca pérdida de intestino y puede combinarse con el aumento vesical usando parte del mismo segmento intestinal aislado ${ }^{27}$.

El objetivo primordial de este estudio es evaluar el resultado del tratamiento quirúrgico de los niños con diagnostico de vejiga neurogénica, asociada o no a colon neurogénico, comparando, en un periodo de 8 años y medio, las diferentes opciones quirúrgicas y los tejidos utilizados.

En este trabajo, al igual que en las revisiones de la literatura ${ }^{33}$, se encuentra que el mielomeningocele es la enfermedad más frecuente que requiere este tipo de reconstrucción quirúrgica $(46,3 \%)$ seguido de otras malformaciones espinales (12,2\%), malformaciones congénitas $(9,8 \%)$, valvas de la uretra posterior $(12,2 \%)$ y el síndrome urofacial $(9,8 \%)$. Castellan et al. ${ }^{25}$ muestran en un estudio de 135 pacientes con 10 años de seguimiento en el que la principal enfermedad que amerita este tipo de cirugía es la mielodisplasia en el 71\% de los casos, seguida de las malformaciones congénitas en el $17 \%$ de los casos.

Se encontró que el 56,1\% de los pacientes fueron de sexo masculino. La edad promedio a la cual se realizó el procedimiento quirúrgico fue de 10,2 años (rango de 1 a 20 años), similar a lo reportado por otros autores, solo Castellan et $\mathrm{al}^{25}$ reportan un promedio de 13 años.

La serie con mayor tiempo de seguimiento es reportada por Liard et $\mathrm{al}^{23}$, con un promedio de 20 años (rango de 15 a 23 años); Castellan et al. ${ }^{25}$ mostró un promedio de seguimiento de 46 meses para el Mitrofanoff; Wedderburn et $\mathrm{al}^{19}$ reporta en una serie de 46 pacientes un seguimiento promedio de 44 meses; nuestro promedio de seguimiento es de 27,2 meses (rango de 1 a 72 meses) lo cual lo hace valioso en la evaluación de resultados a largo plazo.

El estudio de Liard et al. ${ }^{23}$ reporta complicaciones tales como deterioro en la función renal en 10 pacientes, lo cual obligó a realizar otros procedimientos de derivación; reflujo vesicoureteral en 5 pacientes; litiasis vesical en 5 pacientes y obstrucción intestinal en otros 5 pacientes. En nuestro estudio 4 pacientes presentaron formación de cálculos y ninguno presento deterioro de la función renal posterior a la cirugía. 
Encontramos que el 85\% de los niños presentaron infección urinaria antes del procedimiento quirúrgico, con una recurrencia del $75 \%$ después del mismo, sin ningún factor asociado a esta recurrencia, aunque probablemente se deba a una mala técnica en el cateterismo intermitente.

El aumento vesical se realizo en el 68,3\% de los pacientes y el tejido utilizado fue íleon en el 71,4\%, uréter en el $25 \%$ y colon en el 3,6\%, semejante a lo referido en la literatura.

Una opción quirúrgica que mejora la continencia del cuello vesical hasta en un $75 \%$ de los casos, es la construcción de una cincha uretral; la frecuencia global de realización de esta técnica se encuentra alrededor del $15 \%$ y se ha reportado continencia entre el $69 \%$ y el $85 \%$ de los casos a largo plazo ${ }^{13}$. En nuestra institución se realizaron procedimientos sobre el cuello vesical en 21 pacientes $(51,2 \%)$, la mayoría con diagnostico de mielomeningocele y otras malformaciones espinales. Este procedimiento requirió revisión en 4 pacientes (19\%) y 2 pacientes con cincha uretral debieron ser convertidos a cerclaje de cuello. Al momento de la recolección de la información solo un paciente con procedimiento en el cuello presenta incontinencia uretral. Lo anterior describe en nuestro medio resultados, con cerclaje de cuello, semejantes a los reportados en la literatura. En nuestra institución no se dispone de esfínter artificial, método que en el mundo permite una continencia que fluctúa entre el $80 \%$ y $100 \%{ }^{13}$.

La estenosis del estoma vesicocutáneo es la complicación más frecuente del mismo. Los reportes de complicaciones estomales con apéndice varían entre el 19\% y el 26\% $(29,30)$. En este estudio se encontró estenosis del conducto vesicocutáneo cateterizable en el $12 \%$ de los realizados con apéndice y en el $30 \%$ de las realizados con íleon (tubo de Monti). La estenosis del estoma colocutáneo (Malone) se presento en el 8,3\%. Además, se realizo algún tipo de procedimiento sobre el conducto vesicocutáneo por incontinencia urinaria en 8 pacientes (20\%).

De 13 pacientes a quienes se les realizo un conducto colocutáneo cateterizable de Malone, 12 lograron un control fecal adecuado con las irrigaciones colónicas anterogradas. Debe aclararse que el Malone no mejora la continencia fecal, sino que permite mantener el colon limpio mediante los enemas, con el fin de facilitar la evacuación fecal en el momento y el lugar adecuado, mejorando la adaptación social del niño; sin embargo cualquier alteración en la motilidad intestinal puede trastornar dicha evacuación. Los resultados obtenidos con el procedimiento son semejantes a los reportados en la literatura y son satisfactorios para nosotros.

No se encontró ningún reporte que informe acerca de la aceptación del cateterismo por parte del paciente lo cual no permite hacer comparaciones con este trabajo, en el que encontramos una aceptación adecuada del cateterismo, puesto que la mayoría de los problemas con el cateterismo limpio intermitente son secundarios a complicaciones del conducto y no a conductas negligentes de los padres o del niño.

Tekant et al. ${ }^{31}$ utilizaron posteriormente a la cirugía la escala de autoestima de Rosenberg en sus pacientes y aunque no reportan el porcentaje exacto de mejoría si afirman un significativo aumento en la autoestima y disminución en la frecuencia de síntomas depresivos de los pacientes. En nuestro trabajo se intentó una valoración adaptativa según la socialización y escolaridad de los niños de acuerdo con su edad y condición mental, se logró adecuada información de 35 niños, de los cuales 33 realizan actividades escolares y sociales normales. Solo dos niños no asisten a la escuela ni realizan actividades que impliquen interacción social y de éstos uno tiene incontinencia urinaria y mal control fecal (es a quien más procedimientos adicionales se le han realizado). De los niños con adecuada escolaridad e interacción social dos presentan incontinencia urinaria franca y 5 tienen incontinencia urinaria parcial.

Aunque el diseño del estudio es descriptivo, permite sugerir cierta tendencia a que existe una relación entre el resultado en términos de continencia y el proceso de desarrollo del niño, ya que vemos que nuestros pacientes mejoran en forma importante su proceso social cuando logran continencia y pueden acceder mejor a los procesos educativos, puesto que evitan accidentes urinarios y fecales que les generan vergüenza frente a otros niños.

La elección del tipo de conducto a utilizar depende de varios factores que incluyen su disponibilidad, tipo de cirugía y diagnóstico de base ${ }^{32}$. Concluimos que el apéndice es el tejido de elección siempre que pueda ser utilizado, ya que ofrece ventajas técnicas y de resultados sobre los otros tejidos. El uso del tubo de Monti debe condicionarse a la disponibilidad del apéndice, el cual está descrito en el trabajo de Leslie y colaboradores, en el que describen un método predecible, reproducible y duradero para crear un canal continente ${ }^{32}$. Una alternativa es la elaboración del Malone con un colgajo de ciego, lo cual permitiría construir el Mitrofanoff con apéndice en la mayoría de los casos. 
El manejo quirúrgico de la vejiga neurogénica puede prevenir el daño renal y en nuestro caso el aumento vesical presento baja morbilidad ${ }^{33}$; tiene ventajas en términos de continencia, comodidad y calidad de vida para los pacientes. Consideramos que la patología que más riesgo genera para la morbilidad del procedimiento y pronóstico a largo plazo del niño es la vejiga neurogénica de origen medular, ya que son las que más se asocian a otras condiciones patológicas y limitaciones físicas.

El manejo de la vejiga neurogénica exige una valoración completa de las condiciones del paciente, diagnostico de base y patologías asociadas. Los estomas continentes cateterizables son métodos posibles y técnicamente seguros para el tratamiento de la incontinencia urinaria y fecal ${ }^{34}$. No se encontraron diferencias significativas en términos de resultado a largo plazo entre el uso de los diferentes tejidos para elaboración del conducto vesicocutàneo, pero se identificó que los estomas de los conductos realizados con un segmento intestinal tubularizado tuvieron mayores complicaciones que los de los conductos realizados con apéndice, por lo cual el apéndice debe ser el tejido de elección, siempre que esté disponible.

En general los resultados en continencia urinaria, continencia fecal y mejoramiento de la calidad de vida de los niños con vejiga neuropática, son considerablemente mejores a los de aquellos con vejiga neurogénica secundaria a espina bífida o trauma raquimedular.

\section{REFERENCIAS}

1. Schulman SL, Duckett JW. Disorders of bladder function. O’Neill JA, Rowe MI, Grosfeld JL, Fonkalsrud EW, Coran AG, editors. Pediatric surgery, fifth edition, Mosby 1998.

2. Sheldon CA, Snyder HM. Structural disorders of the bladder, augmentation. O'Neill JA, Rowe MI, Grosfeld JL, Fonkalsrud EW, Coran AG, editors. Pediatric surgery, fifth edition, Mosby 1998.

3. Madersbacher H. Neurogenic bladder dysfunction in patients with myelomeningocele. Curr Opin Urol. 2002;12(6):469-472.

4. Aslan AR, Kogan BA. Conservative management in neurogenic bladder dysfunction. Curr Opin Urol. 2002;12(6):473-477.

5. Mills RD, Studer UE. Metabolic consequences of continent urinary diversion. J Urol. 1999;161(4):1057-1066.

6. Mitrofanoff $P$. Trans-appendicular continent cystostomy in the management of the neurogenic bladder. Chir Pediatr. 1980;21(4):297-305.

7. Cain MP, Casale AJ, King SJ, Rink RC. Apendicovesicostomy and new alternatives for the Mitrofanoff procedure: results in the last 100 patients at riley children's hospital. J Urol. 1999;162(5):1749-1752.

8. Gosalbez R, Wei D, Gousse A, Castellan M, Labbie A.. Refashioned short bowel segments for the construction of catheterizable channels (the Monti procedure): early clinical experience. J Urol. 1998;160(3 Pt 2):1099-1102.

9. Hanna MK, Richter F, Stock JA. Salvage continent vesicostomy after enterocystoplasty in the absence of appendix. J Urol. 1999;162(3 Pt 1):826-828.

10. Boemers TM. Mitrofanoff procedure with Meckel's diverticulum. BJU International. 2001;88(7):799-800.

11. Monti PR, Lara RC, Dutra MA, de Carvalho JR. New techniques for construction of efferent conduits based on the Mitrofanoff principle. Urology. 1997;49(1):112-115

12. Van Savage JG, Yepuri JN. Transverse retubularized sigmoidovesicostomy continent urinary diversion to the umbilicus. J Urol. 2001;166(2):644-647.
13. Kryger JV, González R, Barthold JS.. Surgical management of urinary incontinence in children with neurogenic sphincteric incompetence. J Urol 2000;163(1):256-263.

14. Yang WH. Yang needle tunneling technique in creating antrireflux and continent mechanisms. J Urol. 1993;150(3):830-834.

15. Cromie WJ, Barada JH, Weingarten JL. Cecal tubularization: lengthening technique for creation of catheterizable conduit. Urology. 1991;37(1):41-42.

16. Adams MC, Bihrle R, Foster RS, Brito CG. Conversion of an ileal conduit to a continent catheterizable stoma. J Urol. 1992;147(1):126-128.

17. Figueroa TE, Sabogal L, Helal M, Lockhart JL. The tapered and reimplanted small bowel as a variation of the Mitrofanoff procedure: preliminary results. J Urol. 1994;152(1):73-75.

18. Malone PS, Ransley PG, Kiely EM. Preliminary report: the antegrade continence enema. Lancet. 1990;336(8725):1217-1218.

19. Wedderburn A, Lee RS, Denny A, Steinbrecher HA, Koyle MA, Malone PS. Synchronous bladder reconstruction and antegrade continence enema. J Urol. 2001;165(6 Pt 2):2392-2393.

20. Bau MO, Younes S, Aupy A, Bernuy M, Rouffet MJ, Yepremian D, et al. The Malone antegrade colonic enema isolated or associated with urological incontinence procedure: evaluation from patient point of view. J Urol. 2001;165(6 Pt 2):2399-2403.

21. Gerharz EW, et al. The in situ appendix in the Malone antegrade continence enema procedure for faecal incontinence. BJU. 79:985. 1997.

22. Harris CF, et al. Appendicovesicostomy: the Mitrofanoff procedure - A 15 year perspective. J Urol. 2000;163:1922-1926.

23. Liard A, et al. The Mitrofanoff procedure: 20 years later. J Urol. 2001;165: 2394-2398.

24. Kajbafzadeh AM, Chuback N. Simultaneous Malone antegrade continent enema and Mitrofanoff principle using the divided appendix: report of a new technique for prevention of stoma complications. J Urol. 2001;165:24042409.

25. Castellan MA, Gosalbez R, Labbie A, Ibrahim E, DiSandro M. Outcomes of continent catheterizable stomas for urinary and fecal incontinence: comparison among different tissue options. BJU International. 2005;95:1053 1057.

26. Roberts JP, Moon S, Malone PS. Treatment of neuropathic urinary and faecal incontinence with synchronous bladder reconstruction and the antegrade continence enema procedure. BJU. 1995;75:386-389.

27. Castellan MA, Gosalbez R, Labbie A, Monti PR. Clinical applications of the Monti procedures as a continent catheterizable stoma. Urology. 1999;54: 152-156.

28. De Ganck J, Evaraert K, Van Laecke E, Oosterlinck W, Hoebeke PA. A high easy to treat complications rate is a prize for a continent stoma. BJU International. 2002;90:240-243.

29. Narayanaswamy B, Wilcox DT, Cuckow PM, Duffy PG, Ransley PG. The Yang-Monti ileovesicostomy: a problematic channel? BJU International. 2001;87:861-865.

30. Clark T, Pope JC, Adams MC, Wells N, Brock JW. Factors that influence outcomes of the Mitrofanoff and Malone antegrade continence enema reconstructive procedure in children. J Urol. 2002;168:1537-1540.

31. Tekant G, Emir H, Eroglu E, Esenturk N, Buyukunal C, Danismend N, Soylet Y. Catheterizable continent urinary diversion (Mitrofanoff principle) clinical experience and psychological aspects. Eur J Pediatr Surg. 2001;11 (4):263- 267.

32. Leslie JA, Dussinger AM, Meldrum KK. Creation of continence mechanisms (Mitrofanoff) without appendix: the Monti and spiral Monti procedures. Urol Oncol. 2007;25(2):148-153.

33. Proesmans W. The neurogenic bladder: introducing four contributions Pediatr Nephrol. 2008;23(4):537-540.

34. Sultan S, Hussain I, Ahmed B, Aba Umer S, Saulat S, Naqvi SA, et al. Clean intermittent catheterization in children through a continent catheterizable channel: a developing country experience. J Urol. 2008;180(4 Suppl):18521855; discussion 1855

Correspondencia autor: Dra. María E. Arango Rave

Departamento de Cirugía Pediátrica

Universidad de Antioquía. Tel.: 2637867

E-mail autor: rafael195@une.net.co

Información artículo: Original - Vejiga neurógena

Trabajo recibido: marzo 2008

Trabajo aceptado: septiembre 2008 